Acta Crystallographica Section A

Foundations of

Crystallography

ISSN 0108-7673

Received 23 September 1999

(C) 2000 International Union of Crystallography Printed in Great Britain - all rights reserved

\section{Symmetry Elements in Space Groups and Point Groups. Addenda to two IUCr Reports on the Nomenclature of Symmetry ${ }^{1}$}

\author{
H. D. Flack, ${ }^{a *}+$ H. Wondratschek, ${ }^{b}$ Th. Hahn ${ }^{c}$ and S. C. Abrahams ${ }^{d} \ddagger$ \\ aLaboratoire de Cristallographie, Université de Genève, 24 quai Ernest-Ansermet, $\mathrm{CH}-1211$ Genève \\ 4, Switzerland, ${ }^{\mathbf{b}}$ Institut für Kristallographie, Universität Karlsruhe, D-76128 Karlsruhe, Germany, \\ 'Institut für Kristallographie, RWTH Aachen, D-52056 Aachen, Germany, and Physics Depart- \\ ment, Southern Oregon University, Ashland, OR 97520, USA. Correspondence e-mail: \\ howard.flack@cryst.unige.ch
}

The definition of 'symmetry element' given in the Report of the IUCr Ad-Hoc Committee on the Nomenclature of Symmetry by de Wolff et al. [Acta Cryst. (1989). A45, 494-499] is shown to contain an ambiguity in the case of space groups $P 6 / \mathrm{m}, P 6 / \mathrm{mmm}, P 6 / \mathrm{mcc}$ and point groups $6 / \mathrm{m}$ and $6 / \mathrm{mmm}$. The ambiguity is removed by redefining the 'geometric element' as a labelled geometric item in which the label is related to the rotation angle of the rotation or rotoinversion symmetry operation. The complete set of different types of glide plane is shown to contain three more than the 15 that are illustrated in the 1992 Report by de Wolff et al. [Acta Cryst. (1992). A48, 727-732].

\section{Introduction}

The IUCr Ad-Hoc Committee on the Nomenclature of Symmetry presented a Report in 1989 on the definition of symmetry elements in three dimensions (de Wolff et al., 1989), and another in 1992 on the corresponding symbols for symmetry elements and symmetry operations (de Wolff et al., 1992). Pacheco (1998) noticed that Fig. 3 in the 1992 Report, which aimed to present all possible glide-plane aspects, did not contain the diagram for an $n$-glide plane of the $t p$ (tetragonal primitive) Bravais-lattice type. Flack (1998) pointed out that a strict application of the definitions in the 1989 Report to point group $6 / \mathrm{m}$ led to an undefined type of symmetry element. Both matters were referred to readily available members of the original Committee who agreed to investigate the two problems.

\section{Definition of symmetry element}

\subsection{Definition in 1989 Report}

The term symmetry element, widely used over a long period in the crystallographic literature prior to 1989, had been subject to application in a variety of different ways. The Report of 1989 presented a careful definition of this term in order to clarifv its meaning.

\section{$\dagger$ Chair.}

\$E officio.

${ }^{1}$ Established 28 August 1998 as an Ad-Hoc Group by Chair of the Commission on Crystallographic Nomenclature. The Report was received by the Commission 13 July 1999 and accepted 17 September 1999.
The 1989 Report first defined the geometric element by starting from a symmetry operation of a space group or point group. A reduced symmetry operation may be formed from the symmetry operation. For a screw rotation, this is the corresponding rotation; for a glide reflection, it is the corresponding reflection. A geometric element (and thus a symmetry element) is not defined for the identity mapping or the translations. The geometric element is thus the geometric item that allows the reduced symmetry operation to be located and oriented in space. This geometric element is a plane for reflections and glide reflections (mirror plane or glide plane), a line for rotations and screw rotations (rotation axis or screw axis), a point for inversions (centre of inversion), and a point on a line for rotoinversions (rotoinversion axis), see Tables 1 and 2 of the 1989 Report. These tables are repeated in the 1992 Report; an omitted comma after 'Angle and sense of rotation', under 'Additional parameters' for screw rotation operations in Table 1 of 1992, is noted to avoid the possibility of confusion.

Different symmetry operations of a symmetry group or crystal may have the same geometric element. Therefore, the element set of a geometric element is defined as the set of all symmetry operations of a space group or point group which have the same geometric element, see Tables 1 and 2 of the 1989 Report. The symmetry element is the combination of the geometric element with the element set. Its name is formed by attaching the symbol of the element set to the geometric element; for example, a twofold rotation axis, a threefold screw axis, a glide plane, a fourfold rotoinversion axis etc. The designation of a symmetry element, however, is derived from 


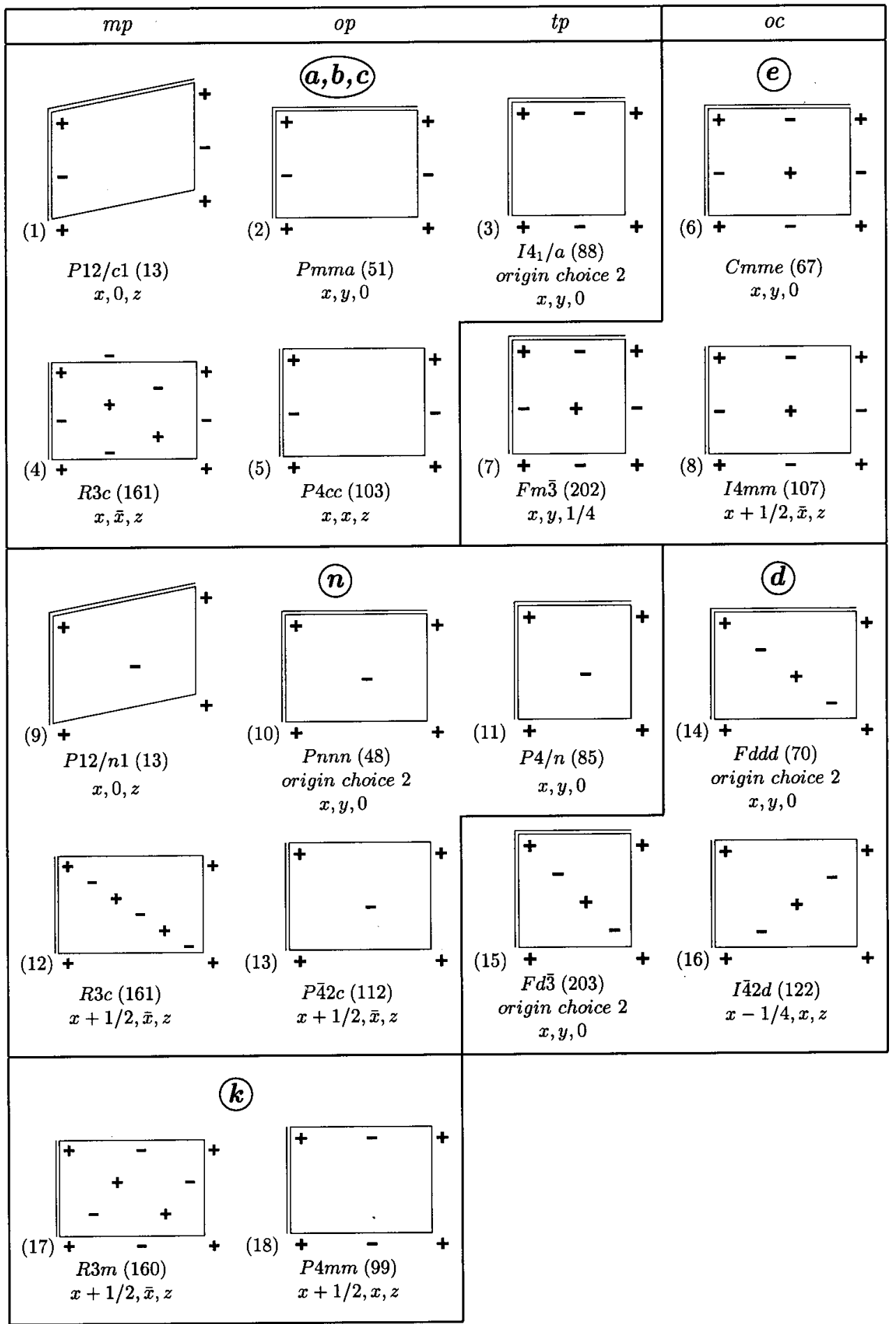

Figure 1

This figure replaces Fig. 3 in the Report on the Nomenclature of Symmetry by de Wolff et al. (1992), with three diagrams (7), (11) and (15) included that previously were missing, see $\$ 3$. The different kinds of glide plane are represented graphically by diagrams similar to the general-position diagrams in ITA. The origin is always in the upper left corner, with symmetry operations represented by a '+' for translations, a '-' for glide reflections. Double lines represent crystal axes in the plane of the figure, single lines other cell edges. Each diagram contains a space-group example with glide-plane location listed. The setting is conventional in all diagrams with two crystal axes as cell edges; it is unconventional in all other cases. The diagrams are presented in columns of Bravais net types of the section plane; the unconventional $c$-centred setting of the square net in diagrams (7) and (15) is the result of a section parallel to (001) through a conventional cubic $F$ lattice. The vertical edge of the cell (in the plane of the paper) represents: (i) the $c$ axis in diagrams (1), (4), (5), (8), (9), (12), (13), (16), (17), (18); (ii) the $a$ axis in diagrams (2), (3), (6), (7), (10), (11), (14), (15). The horizontal edge of the cell represents: (i) the $a$ axis in diagrams (1), (9); (ii) the [1̄10] direction in diagrams (5), (16), (18); (iii) the [110] direction in diagrams (4), (8), (12), (13), (17); (iv) the $b$ axis in diagrams (2), (3), (6), (7), (10), (11), (14), (15). 
the complete element set of the geometric element, e.g. the symmetry element of a threefold screw rotation with axis through the origin in space group $P 3_{1}$ is a threefold screw axis, in space group $P 6_{1}$ it is a sixfold screw axis. It is noted that further specification of these symmetry elements is possible only if the crystal structure (the space group) and thus its translation lattice is known. The combination of a counterclockwise rotation by $120^{\circ}$ about the direction [111] with a lattice translation by a, i.e. the symmetry operation $z+1, x, y$ [in the notation of International Tables for Crystallography (1996), Vol. A, hereafter ITA], of the cubic space group P23, is a threefold screw rotation. Its symmetry element is a $3_{1}$ screw axis through the point $\frac{2}{3}, \frac{1}{3}, 0$ in space group $P 23$, the symmetry element of the same symmetry operation is a $3_{2}$ screw axis in space group $I 23$. The symmetry element of the glide reflection $x+\frac{1}{2}, y, \bar{z}$ is an $a$-glide plane in space group Pmma but an $e$-glide plane in space group Cmme (formerly Cmma).

\subsection{Recommended definition}

The definition of 'symmetry element' in $\$ 2.1$ breaks down in space groups $P 6 / \mathrm{m}, P 6 / \mathrm{mmm}, P 6 / \mathrm{mcc}$ and point groups $6 / \mathrm{m}$ and $6 / \mathrm{mmm}$, as observed by Flack (1998). The geometric element of the rotoinversion $\overline{6}$ in site symmetry $6 / m$ is a line with a point defined on it. However, not only $\overline{6}$ and $\bar{\sigma}^{-1}$ (the inverse of $\overline{6}$ ) but also $\overline{3}$ and $\overline{3}^{-1}$ belong to this geometric element. The second sentence of the second paragraph in $\$ 5$ of the 1989 Report: 'Given that geometric element, it is the element set (consisting of the operations sharing that geometric element) that determines the nature of (and, eventually, the symbol for) the symmetry element. There is always just one such set, so that no ambiguity can exist and it is only the symbol which may be open to discussion' hence does not hold in this example.

The symmetry element concept in the Report of 1989 is so clear and useful that it should be retained. However, the inconsistency in the last paragraph should be removed. This is easily accomplished by defining the geometric element not as a purely geometric item (point, line, plane, line + point on it) but as a labelled geometric item. The geometric item is marked by an appropriate designation of the original (defining) operation. The geometric element of the $\overline{6}$ operation in $6 / m$ is not line + point but, rather, is line + point coupled to $\overline{6}$. Thus defined, $\overline{3}$ and $\overline{3}^{-1}$ do not belong to the element set of this geometric element because its element set is only $\overline{6}$ and $\overline{6}^{-1}$, see Tables 1 and 2 in the 1989 Report. The resulting ambiguity has been lifted by recalling the original (defining) symmetry operation. For this reason, the definition of the geometric element is augmented by specifying a label related to the rotation angle of the rotation or rotoinversion symmetry operation.

It is noteworthy that such difficulties do not arise in space groups $P 6_{3} / \mathrm{m}, P 6_{3} / \mathrm{mcm}$, and $P 6_{3} / \mathrm{mmc}$ due to the separation of invariant points in $\overline{6}$ and $\overline{3}$.

\section{Types of glide plane}

The list of 15 types of glide plane in Fig. 3 of the 1992 Report is incomplete, as originally discovered by Pacheco (1998) who suggested the addition of a diagram such as (11) in the present Fig. 1. Two further omissions, displayed as diagrams (7) and (15) in the present Fig. 1, were discovered during this study by H. Wondratschek. Fig. 3 of the 1992 Report should hence be replaced by the present Fig. 1 . It should be noted that the numbers of some diagrams in the present Fig. 1 are changed with respect to their numbering in Fig. 3 of the 1992 Report. This is necessary to place the new diagrams in the most appropriate sites.

It is also noted that the first term $o c$ in the final column of Table 3 of the 1992 Report should be changed to $o c, t p(t c)$. The special orientation of the tetragonal net should be indicated because the setting of the tetragonal net is centred in cubic space groups with lattice type $F$, see also the present Fig. 1.

Appreciation is expressed to Mr J. V. Pacheco for bringing the omission in Fig. 3 of the 1992 Report to our attention. The help of Ralf Müller, Karlsruhe, Germany, in drawing Fig. 1 is gratefully acknowledged.

\section{References}

Flack, H. D. (1998). Private communication.

International Tables for Crystallography (1996). Vol. A, Space-Group Symmetry, 4th revised ed., edited by Th. Hahn. Dordrecht: Kluwer Academic Publishers.

Pacheco, J. V. (1998). Private communication.

Wolff, P. M. de, Billiet, Y., Donnay, J. D. H., Fischer, W., Galiulin, R. B., Glazer, A. M., Hahn, Th., Senechal, M., Shoemaker, D. P., Wondratschek, H., Wilson, A. J. C. \& Abrahams, S. C. (1992). Acta Cryst. A48, 727-732.

Wolff, P. M. de, Billiet, Y., Donnay, J. D. H., Fischer, W., Galiulin, R. B., Glazer, A. M., Senechal, M., Shoemaker, D. P., Wondratschek, H., Hahn, Th., Wilson, A. J. C. \& Abrahams, S. C. (1989). Acta Cryst. A45, 494-499. 